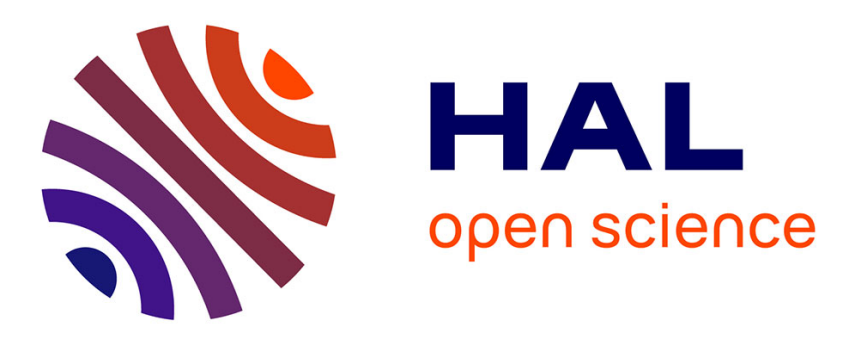

\title{
Detection of electromagnetic multipoles by x-ray spectroscopies
}

\author{
Sergio Di Matteo, Y. Joly, C. R. Natoli
}

\section{To cite this version:}

Sergio Di Matteo, Y. Joly, C. R. Natoli. Detection of electromagnetic multipoles by x-ray spectroscopies. Physical Review B: Condensed Matter and Materials Physics (1998-2015), 2005, 72 (14), pp.144406. 10.1103/PhysRevB.72.144406 . hal-01533960

\section{HAL Id: hal-01533960 https://hal.science/hal-01533960}

Submitted on 6 Jun 2017

HAL is a multi-disciplinary open access archive for the deposit and dissemination of scientific research documents, whether they are published or not. The documents may come from teaching and research institutions in France or abroad, or from public or private research centers.
L'archive ouverte pluridisciplinaire HAL, est destinée au dépôt et à la diffusion de documents scientifiques de niveau recherche, publiés ou non, émanant des établissements d'enseignement et de recherche français ou étrangers, des laboratoires publics ou privés. 


\title{
Detection of electromagnetic multipoles by resonant x-ray spectroscopies.
}

\author{
S. Di Matteo ${ }^{1,2}$, Y. Joly ${ }^{3}$, C. R. Natoli ${ }^{1}$ \\ ${ }^{1}$ Laboratori Nazionali di Frascati INFN, via E. Fermi 40 I-00044 Frascati (Roma) Italy \\ ${ }^{2}$ Dipartimento di Fisica "E. Amaldi", Università di Roma 3, via della Vasca Navale 84, I-00146 Roma, Italy \\ ${ }^{3}$ Laboratoire de cristallographie, CNRS, BP166, F-38042 Grenoble Cedex 9, France
}

(August 12, 2005)

\begin{abstract}
Following a brief discussion on electromagnetic multipole expansion, we provide a link between several multipole moments and the measurable quantities of some x-ray spectroscopies, like resonant x-ray scattering (RXS) or dichroism in absorption. A general classification is given, accompanied by some specific examples and a geometrical interpretation of the axial toroidal (non-magnetic) quadrupole, related to the x-ray natural circular dichroism. Finally, in order to provide a physical example for the detection of magnetic parity-odd multipoles, like the toroidal moment, we perform a numerical simulation for x-ray non-reciprocal directional dichroism, at Fe $\mathrm{K}$ edge, in the polar ferrimagnet $\mathrm{GaFeO}_{3}$. Our calculations describe quite well the reported experimental profile, with the correct order of magnitude for dichroism/absorption intensity-ratio and a signal which is limited to pre-edge.
\end{abstract}

PACS numbers: $78.70 . \mathrm{Ck}, 71.30 .+\mathrm{h}$

\section{INTRODUCTION}

Multipole expansion of electric and magnetic fields generated by fixed charges and permanent currents is a widely used tool to characterize the electromagnetic state of a physical system. ${ }^{1}$ For example, for a charge distribution $\rho(\vec{x})$ in a given external potential field $\Phi(\vec{x})$, whose electrostatic energy is $W_{E}=\int d^{3} x \rho(\vec{x}) \Phi(\vec{x})$, one can exploit the well-known expansion: $W_{E}=q \Phi(0)-\vec{d} \cdot \vec{E}-$ $\frac{1}{6} \sum_{i j} Q_{i j} \frac{\partial E_{i}}{\partial x_{j}}(0)+\ldots$. Here $(0)$ is some properly chosen origin, and $\vec{E}=-\vec{\nabla} \Phi$. Such an expansion shows how the external field couples with the various multipoles of the charge distribution: the potential with the charge $q$, the electric field with the dipole $\vec{d}$, the gradient of the field with the quadrupole $Q_{i j} \equiv 3 x_{i} x_{j}-r^{2} \delta_{i j}$, and so on. A similar expansion holds also for the vector potential. In this case there is no monopole term, of course, and the magnetic energy is: $W_{M}=\int d^{3} x \vec{J}(\vec{x}) \cdot \vec{A}(\vec{x})$. However, due to the vector character of the current density $\vec{J}$ and the potential $\vec{A}$, it is often useful to decompose them, before exploiting the expansion, in a longitudinal (rotor-free) and a transversal (divergenceless) part: $\vec{J}=\vec{J}_{\|}+J_{\perp}$. As $\vec{\nabla} \cdot \vec{J}=\vec{\nabla} \cdot \vec{J}_{\|} \equiv-\partial_{t} \rho$, then the longitudinal $\vec{J}_{\|}$is related to the time-derivative of the charge multipoles. On the other side, the solenoidal field $\vec{J}_{\perp}$ is characterized by two independent families of moments, and can be written, in the Helmholtz-Debye representation, as: $\vec{J}_{\perp}=\vec{l} \psi(\vec{x})+\vec{\nabla} \times(\vec{l} \chi(\vec{x}))$, where $\vec{l}$ is the orbital angular momentum and $\psi(\vec{x})$ and $\chi(\vec{x})$ are a scalar and pseudoscalar function, respectively. The first vector describes the toroidal currents (flowing along the parallels on a sphere), and the second the poloidal currents (flowing along the meridians on a torus). Toroidal currents are widely used, eg, in the physics of electron plasma. Following Ref. 2, we define the magnetic multipoles of the two families in terms of the orbital angular momentum; when also spin quantities play a role, it is sufficient to consider the substitution $\vec{l} \rightarrow \vec{l}+g_{s} \vec{s}$, where $g_{s}$ is the spin giromagnetic ratio. Then we have:

a) Magnetic moment: $\vec{m}=\frac{e}{2 m} \vec{l}=\mu_{B} \vec{l}$;

b) Magnetic quadrupole: $m_{i j}=\mu_{B}\left(x_{i} l_{j}+l_{i} x_{j}\right)$;

c) Magnetic (polar) toroidal moment: $\vec{t}=\oint(\vec{x} \times \vec{m}) d^{3} x$;

d) Magnetic toroidal quadrupole: $t_{i j}=x_{i} t_{j}+t_{i} x_{j}$.

Notice that the magnetic toroidal moment is known in high-energy physics as anapole. ${ }^{3} \mathrm{~A}$ simple geometrical image of $\vec{t}$ is a closed circle of elementary magnets $\vec{m}$ joined to each other. By analogy with this image, one can define a similar quantity, ${ }^{4}$ by replacing $\vec{m}$ with the electric dipole $\vec{d}$. Then we get:

e) Axial toroidal moment: $\vec{g}=\oint(\vec{x} \times \vec{d}) d^{3} x$;

f) Axial toroidal quadrupole: $g_{i j}=x_{i} g_{j}+g_{i} x_{j}$.

These moments are scalarly coupled with the corresponding fields in the following way: $\vec{m}$ and $m_{i j}$ couple, respectively, with the magnetic field $\vec{B}$ and its gradient $\partial_{i} B_{j}$; magnetic toroidal moment $\vec{t}$ and quadrupole $t_{i j}$ couple, respectively, with the time-reversal $(\hat{T})$ odd and inversion $(\hat{P})$ odd vector $\vec{E} \times \vec{B}$ and its $\hat{P}$-even gradient $\partial_{i}(\vec{E} \times \vec{B})_{j}$; finally, axial toroidal moment $\vec{g}$ and quadrupole $g_{i j}$ couple with the $\hat{T}$-even and $\hat{P}$-even vector $\vec{E} \times \vec{B}(\vec{E} \cdot \vec{B})$ and its $\hat{P}$-odd gradient $\partial_{i}(\vec{E} \times \vec{B})_{j}(\vec{E}$. $\vec{B})$. In order to have non-zero toroidal moments and quadrupoles, the symmetry of the crystal must be subject to special conditions: in fact, c) is $\hat{T}$ and $\hat{P}$-odd, $d)$ is $\hat{T}$ odd and $\hat{P}$-even, $e$ ) is $\hat{T}$ and $\hat{P}$-even and $f)$ is $\hat{T}$-even and $\hat{P}$-odd. Remarkably, in a polarized system, the interaction of the dipole $\vec{g}$ with the fields $\vec{E}, \vec{B}$ is possible also at a lower order and in the absence of a net magnetization: the corresponding free-energy is $F \propto \vec{g} \cdot(\vec{D} \times \vec{E})$, where $\vec{D}$ is the (local or global) polarization of the system. ${ }^{2}$ The knowledge of electric and magnetic multipoles of both 
parities under space-inversion and time-reversal can be of great importance in order to understand the electromagnetic behaviour of the material: the possibility to use any of these multipoles as an order parameter in phase transitions has been analyzed, eg, in Ref. [2].

The aim of this paper is to show how it is possible to detect most of the previous multipoles by means of resonant x-ray spectroscopies. In section II we shall explicitly write the one-to-one correspondance of each multipole with the measurable quantities in resonant $\mathrm{x}$-ray scattering (RXS) and dichroism in absorption. In particular, the results of x-ray natural circular dichroism $(\mathrm{XNCD})^{5}$ is interpreted as a measure of the non-magnetic toroidal quadrupole of the system, $g_{i j}$, contrary to what presently believed. ${ }^{6}$ Finally, in section III we perform a new calculation to describe the results of a recent nonreciprocal dichroism experiment, called x-ray directional dichroism, in the polar ferrimagnet $\mathrm{GaFeO}_{3} .{ }^{7}$ The physical quantities involved in such a dichroism are shown to be a mixture of magnetic quadrupole, toroidal magnetic moment and octupole, ie, quantities resulting from dipole-quadrupole (E1-E2) interference which are parity and time-reversal odd.

\section{MULTIPOLAR TERMS IN RXS AND ABSORPTION.}

Core resonant spectroscopies are described by the virtual processes that promote a core electron to some empty energy levels. They all depend on the transition matrix elements of matter-radiation interaction:

$$
M_{n g}^{i(o)}(j)=\left\langle\psi_{n}\left|\hat{O}^{i(o)}\right| \psi_{g}(j)\right\rangle
$$

where, in the x-ray regime, the operator $\hat{O}$ is written through the multipolar expansion of the photon field up to electric dipole (E1) and quadrupole (E2) terms ${ }^{8}$ :

$$
\hat{O}^{i(o)}=\vec{\epsilon}^{i(o)} \cdot \vec{r}\left(1-\frac{1}{2} i \vec{k}^{i(o)} \cdot \vec{r}\right)
$$

In Eq. (1), $\psi_{g}(j)$ is the core ground state centered around the $j^{\text {th }}$ atom and $\psi_{n}$ the photo-excited state, whereas in Eq. (2), $\vec{r}$ is the electron position measured from the absorbing ion, $\vec{\epsilon}^{i(o)}$ is the polarization of the incoming (outgoing) photon and $\vec{k}^{i(o)}$ its corresponding wave vector. In RXS the global process of photon absorption, virtual photoelectron excitation and photon reemission, is coherent throughout the crystal, thus giving rise to the usual Bragg diffraction condition:

$$
F=\sum_{j} e^{i \vec{Q} \cdot \vec{R}_{j}}\left(f_{0 j}+f_{j}^{\prime}+i f_{j}^{\prime \prime}\right)
$$

Here $\vec{R}_{j}$ stands for the position of the scattering ion $j$, $\vec{Q}$ is the diffraction vector and $f_{0}$ is the usual Thomson factor. The resonant part, $f_{j}^{\prime}+i f_{j}^{\prime \prime} \equiv f_{j}$, is the anomalous atomic scattering factor, given by the expression ${ }^{9}$ :

$$
f_{j}(\omega)=\frac{m_{e}}{\hbar^{2}} \frac{1}{\hbar \omega} \sum_{n} \frac{\left(E_{n}-E_{g}\right)^{3} M_{n g}^{o *}(j) M_{n g}^{i}(j)}{\hbar \omega-\left(E_{n}-E_{g}\right)-i \frac{\Gamma_{n}}{2}}
$$

where $\hbar \omega$ is the photon energy, $m_{e}$ the electron mass, $E_{g}$ the ground state energy, and $E_{n}$ and $\Gamma_{n}$ are the energy and inverse lifetime of the excited states. The sum is extended over all the excited states of the system.

The connection with x-ray near edge absorption (XANES) is straightforward, as its cross-section $\sigma$ simply corresponds to the imaginary part of $f_{j}(\omega)$ when $\hat{O}^{o}=\hat{O}^{i}$ (forward scattering):

$$
\sigma=-4 \pi \cdot 10^{22} \frac{a_{0}^{2} \alpha^{3} m_{e} c^{2}}{\hbar \omega} f^{\prime \prime}
$$

Here $a_{0}$ is the Bohr radius, $\alpha$ is the fine structure constant, $c$ is the speed of light and the prefactors are such that $\sigma$ is in Mbarn when $f^{\prime \prime}$ is in units of the classical electron radius, $r_{0} \simeq 2.82 \cdot 10^{-15} \mathrm{~m}$.

The matrix element in Eq. (1) depends only on the electronic part of the operator $\hat{O}$, so that the radiation parameters $\hat{\epsilon}$ and $\vec{k}$ can be factorized out. It is then possible to demonstrate ${ }^{10,11}$ that the RXS amplitude, Eq. (4), as well as XANES cross section, Eq. (5), can be expressed as scalar product of two irreducible tensors, $f_{j}(\omega)=\sum_{p, q}(-)^{q} T_{q}^{(p)} F_{-q}^{(p)}(j ; \omega)$, where $T_{q}^{(p)}$ depends only on the incident and scattered polarization and wave vectors, while $F_{q}^{(p)}(j ; \omega)$ represents the properties of the system under study. It is well-known from the literature (see, e.g., Refs. [ 12-14], or Ref. [ 15], for a treatment in cartesian basis) that the rank $p$ of these irreducible tensors depends on the order of the multipoles in the electromagnetic field expansion, Eq. (2): for example, in the E1-E1 channel $p=0,1,2$, in the E1-E2 channel $p=1,2,3$ and in the E2-E2 channel $p=0,1,2,3,4$. Moreover, for each $p, F_{q}^{(p)}(j ; \omega)$ is related to a specific term of the multipolar expansion of the system, according to the following table:

\begin{tabular}{c|ccc} 
tensor & $\hat{T} \hat{P}$ & multipole \\
\hline$F^{(0)}(\mathrm{E} 1-\mathrm{E} 1)$ & + & + & electric charge \\
$F^{(1)}(\mathrm{E} 1-\mathrm{E} 1)$ & -+ & magnetic dipole \\
$F^{(2)}(\mathrm{E} 1-\mathrm{E} 1)$ & ++ & electric quadrupole \\
$F^{(1+)}(\mathrm{E} 1-\mathrm{E} 2)$ & +- & electric dipole \\
$F^{(2+)}(\mathrm{E} 1-\mathrm{E} 2)$ & +- & $\vec{g}-$ quadrupole \\
$F^{(3+)}(\mathrm{E} 1-\mathrm{E} 2)$ & +- & electric octupole \\
$F^{(1-)}(\mathrm{E} 1-\mathrm{E} 2)$ & -- & polar toroidal dipole \\
$F^{(2-)}(\mathrm{E} 1-\mathrm{E} 2)$ & - & - & magnetic quadrupole \\
$F^{(3-)}(\mathrm{E} 1-\mathrm{E} 2)$ & - & - & polar toroidal octupole \\
$F^{(3)}(\mathrm{E} 2-\mathrm{E} 2)$ & - & + & magnetic octupole \\
$F^{(4)}(\mathrm{E} 2-\mathrm{E} 2)$ & + & + & electric hexadecapole
\end{tabular}

For $p=0,1,2, \mathrm{E} 1-\mathrm{E} 1$ and E2-E2 tensors represent the same physical quantities, though referred to states with different angular momentum. The identification illustrated in Table I is dictated by the unique properties 
under time-reversal and parity reflections of $F^{(p)}$ tensors on one side and multipole terms on the other. In fact, for any given tensor rank $p=1,2,3,4$, there is just one electromagnetic multipole of the same rank $(1 \rightarrow$ dipole, $2 \rightarrow$ quadrupole, $3 \rightarrow$ octupole, $4 \rightarrow$ hexadecapole) with the same $\hat{T}$ and $\hat{P}$ properties. Notice that $\hat{P}$-odd E1-E2 tensors have both $\hat{T}$-odd $(-)$ and $\hat{T}$-even $(+)$ terms for any $p,{ }^{14}$ while $\hat{P}$-even tensors (both E1-E1 and E2-E2) are $\hat{T}$-odd if of odd rank and $\hat{T}$-even if of even rank.

As each $F^{(p)}$ is scalarly related to a corresponding $T^{(p)}$, they have the same $\hat{T}$ and $\hat{P}$ properties. For example, in the E1-E1 or E1-E2 channels, $T^{(0)} \equiv \hat{\epsilon}^{o *} \cdot \hat{\epsilon}^{i}$ is a scalar and $T^{(1)} \equiv \hat{\epsilon}^{o *} \times \hat{\epsilon}^{i}$ is a $\hat{T}$-odd and $\hat{P}$-even vector. Analogously, in the E1-E2 channel, the previous quantities combine with the two $\hat{P}$-odd vectors $\vec{k}^{i}+\vec{k}^{o}$, which is $\hat{T}$-odd, and $\vec{k}^{i}-\vec{k}^{o}$, which is $\hat{T}$-even (see, e.g., Ref. [ 16] for a more complete analysis of this case).

Up to now, we have deliberately avoided the question of whether the local density of the tensors probed by these spectroscopy has anything to do with the properties of the ground state, which we are interested in. At first sight, the presence of the core hole in the final state would prone to give a negative answer to this question, and yet, the wide succes of sum rules for magnetic circular dichroism ${ }^{17}$ seems to prove the contrary. Moreover, in Ref. [14] in the framework of multiple scattering theory, it was demonstrated that these spectroscopies are sensitive to the expectation values of some tensor operators in the scattering states at the energy of the measurement, so that, by integrating over a specific edge, sum rules can be recovered. ${ }^{18}$ Thus, in the following we assume that we may refer to the values of the multipoles in the groundstate.

The sum-rule related to $\mathrm{K}$ edge magnetic circular dichroism is well known ${ }^{17}$ : in the E1-E1 channel, the dichroic signal is proportional to the orbital magnetic moment $\vec{l}$ projected along the photon wave vector. Similarly, in the E1-E2 channel, XNCD was found to be sensitive to the mean value of the tensor $F^{(2+)}(\mathrm{E} 1-\mathrm{E} 2) .{ }^{5}$ It is interesting to note that this latter has been interpreted in the past ${ }^{6,11,16}$ as the expectation value of the physical quantity $\left\langle l_{z} t_{z}\right\rangle$, while from the previous table it comes out that this term measures the quadrupole of $\vec{g}$, which is time-reversal even and parity-odd. Quantitatively, the former identification is not incorrect, as the expectation values of the operators $\left\langle l_{z} t_{z}\right\rangle$ and $\left\langle r_{z} g_{z}\right\rangle$ between states with $\Delta l= \pm 1$ are proportional to each other. However, from the physical point of view it seems rather singular that a non-magnetic quantity like XNCD be related to the expectation value of two magnetic terms like $\vec{t}$ and $\vec{l}$. It is now clear that $l_{z} t_{z}$ is nothing but an effective operator representing the $\vec{g}$-quadrupole when averaged between states differing by one unit of angular momentum. Therefore, it is the $\vec{g}$-quadrupole that measures the rotatory power in XNCD and RXS spectroscopies.

A simple geometrical model that may help in the visualization of such a term can be constructed as follows.
Consider any local structure with $\hat{C}_{3}$-symmetry, like $\mathrm{V}^{3+}$ ions in corundum $\mathrm{V}_{2} \mathrm{O}_{3}$ or $\mathrm{I}^{5+}$-ions in $\alpha$ - $\mathrm{LiIO}_{3}$. Such structures can be seen from the three-fold axis as superpositions of a trigonal distortion ( $D_{3 d}$ local symmetry) and a relative three-fold twisting of the oxygens "above" and "below" the cation, as shown in Fig. 1. In particular, Fig. 1(a) represents the untwisted situation, where the local symmetry is not lower than $C_{3 v}$ and no local $F^{(2+)}(\mathrm{E} 1-\mathrm{E} 2)$ term is allowed, as forbidden by the vertical mirror plane (see, eg, Ref. [11]). In Fig. 1(b), the arrows show the local displacement of the oxygens following a rotation about the (111) axis, which reduces the symmetry to $C_{3}$ : if, in first approximation, we consider the $d$-orbitals as frozen, this induces a local polarization around the oxygens as shown by the charge positions in Fig. 1(c). This orbital polarization is in turn equivalent to the electric dipole configuration, drawn in Fig. 1(c) and (d), with a net rotation, oppositely oriented "above" and "below" the cation ion. As depicted in Fig. 1(d) such a configuration is equivalent to a couple of $\vec{g}$-dipoles with opposite direction, in this way creating a quadrupole $g_{i j}$. Notice that when we relax the approximation of frozen orbitals, only an actual calculation can tell us whether the polarization still persists or not: a posteriori this is confirmed by the signals obtained through numerical $a b$ initio simulations in Refs. [5, 11].
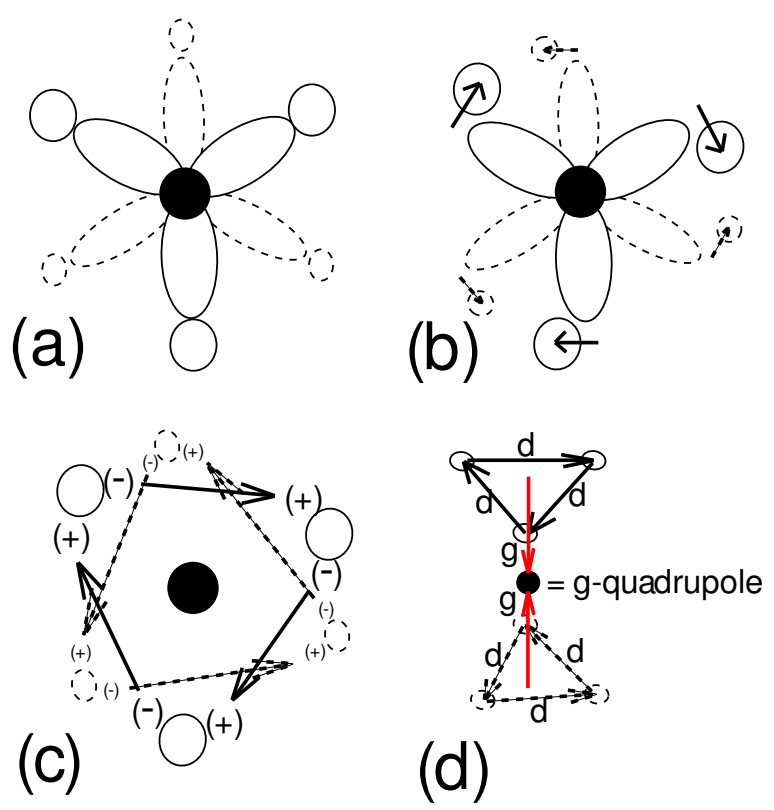

FIG. 1. Schematic description of the formation of the g-quadrupole. The local structure of corundum $\mathrm{V}_{2} \mathrm{O}_{3}$ is considered. The mechanism is explained in the text. The full circle indicates the $\mathrm{V}$-ion and the empty ones represent the oxygens. The (111) direction is perpendicular to the plane of the figure in (a), (b) and (c) and $p$-like orbitals are depicted in (a) and (b). Black arrows in (c) and (d) represent electric dipoles. Dashed objects lie "below" the V-ion plane.

These same quantities are detectable also by means of 
RXS, where the local transition amplitudes are added with a phase factor that can compensate the possible vanishing effect due to the global symmetry, as shown in Ref. [11] for the quadrupole of $\vec{g}$ in the corundum phase of $\mathrm{V}_{2} \mathrm{O}_{3}$ and, e.g., in Ref. [19] for the magnetic moment in the antiferromagnetic insulating phase of $\mathrm{V}_{2} \mathrm{O}_{3}$.

Thus, simply on the basis of their $\hat{P}$ and $\hat{T}$ properties shown in Table I, it follows that three of the four basic vectors $(\vec{d}, \vec{m}$ and $\vec{t})$ are detectable, while $\vec{g}$ is not, as there is no rank-one tensor which is $\hat{P}$ and $\hat{T}$-even. Similarly, it is not possible to detect with these techniques the magnetic toroidal quadrupole $t_{i j}$, as there is no rank-two tensor which is $\hat{P}$-even and $\hat{T}$-odd.

In conclusion, $\mathrm{x}$-ray spectroscopies allow us to gain information about the following set of electromagnetic multipoles (the first five are parity-even, E1-E1 or E2-E2, the latters parity-odd, E1-E2):

1) Charge, expressed by $F^{(0)}$. This is usually the strongest contribution in both XANES and RXS. This term allows us to prove (or disprove) the so-called chargeordering through forbidden diffraction-peaks analysis (see, e.g., Ref. [20]). In fact, any modification of the atomic charge implies a core level shift, and the measure of the energy shift of the threshold allows us to obtain the total valence charge with a good resolution.

2) Magnetic moments (ie, $\left.F^{(1)}(\mathrm{E} 1-\mathrm{E} 1)\right)$ have been treated in a widespread context, due to the big success of the "Carra-Thole-van der Laan" sum-rules. ${ }^{17}$

3) Electric quadrupole $\left(F^{(2)}(\mathrm{E} 1-\mathrm{E} 1)\right)$ is the biggest non-magnetic anisotropic term, responsible, e.g., for structural linear dichroism, as well as, in RXS signals, for the anomalous Jahn-Teller and "orbital ordering" peaks in systems like manganites. ${ }^{21}$

4) Magnetic octupole $\left(F^{(3)}(\mathrm{E} 2-\mathrm{E} 2)\right)$ was invoked in Ref. [ 22] to explain the anomalous RXS signal in $\mathrm{NpO}_{2}$ and was proved to give a contribution in the anomalous magnetic signal in $\mathrm{V}_{2} \mathrm{O}_{3} \cdot{ }^{19,23}$

5) Electric hexadecapole $\left(F^{(4)}(\mathrm{E} 2-\mathrm{E} 2)\right)$ was detected in $\alpha$-haematite by Finkelstein and collaborators, as explained in Ref. [ 10].

6) Electric dipole $\left(F^{(1+)}(\mathrm{E} 1-\mathrm{E} 2)\right)$ is observable by RXS in antiferroelectric materials, like $\mathrm{ErGe}_{3}$ or $\mathrm{VOMOO}_{4} \cdot{ }^{24}$

7) Non-magnetic (axial) toroidal quadrupole, as discussed above, was found with XNCD in $\alpha-\mathrm{LiIO}_{3},{ }^{5}$ and in $\mathrm{V}_{2} \mathrm{O}_{3}$ by means of RXS. ${ }^{11}$

8) Electric octupole $\left(F^{(3+)}(\mathrm{E} 1-\mathrm{E} 2)\right)$ has been predicted in Ref. [16] for $\mathrm{K}_{2} \mathrm{CrO}_{4}$ on the basis of symmetry arguments.

9-10-11) Finally, magnetic toroidal moment $\left(F^{(1-)}(\mathrm{E} 1-\mathrm{E} 2)\right)$ and octupole $\left(F^{(3-)}(\mathrm{E} 1-\mathrm{E} 2)\right)$, together with magnetic quadrupole $\left(F^{(2-)}(\mathrm{E} 1-\mathrm{E} 2)\right)$ have been found in the anomalous magnetic signal of $\mathrm{V}_{2} \mathrm{O}_{3} \cdot{ }^{19}$ Unfortunately, in this latter case all three terms were entangled with the magnetic octupole of E2-E2 origin, and it was not clear how to separate one from the other. The possibility of disentangling the three E1-E2 contribution has been demonstrated in Ref. [ 25] for $\mathrm{Li}_{2} \mathrm{VOSiO}_{4}$ where it was even shown how to extract each single term separately, by means of a series of azimuthal scans. However, this was just a theoretical prediction, not yet confirmed by an experiment. Of course, a much more cogent test to demonstrate the detectability of such quantities is represented by a convergent description of theory and experiment. Such an experiment has already been performed with a dichroic technique in absorption, ${ }^{7}$ and in the next section we shall provide its quantitative interpretation in terms of the three time-reversal and parity-odd quantities $F^{(1-)}(\mathrm{E} 1-\mathrm{E} 2), F^{(2-)}(\mathrm{E} 1-\mathrm{E} 2)$, $F^{(3-)}(\mathrm{E} 1-\mathrm{E} 2)$.

\section{X-RAY NON-RECIPROCAL DIRECTIONAL

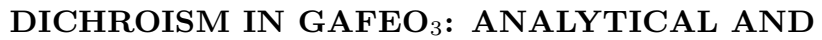 NUMERICAL CALCULATIONS.}

$\mathrm{GaFeO}_{3}$ is a piezoelectric crystal which was first synthetized by J.P. Remeika more than 40 years ago. ${ }^{31}$ Below about $\mathrm{T}_{c} \simeq 200 \mathrm{~K},{ }^{32}$ it also shows a spontaneous magnetization, with magnetic Shubnikov group m'2'm which is characterized by an off-diagonal magnetoelectric susceptibility. A very large linear magnetoelectric effect was reported by Rado. ${ }^{33}$ The presence of both symmetric and antisymmetric parts in the magnetoelectric susceptibility tensor makes possible the detection of both nonreciprocal gyrotropy and magnetochiral effects through x-ray dichroism in absorption (see, e.g., Refs. [ 14, 34]). This possibility was realized in Ref. [7], in which a beautiful experiment was performed under the name of x-ray non-reciprocal directional dichroism (XNDD) whereby a time-reversal and parity-odd signal was detected. Even though we basically agree with the general interpretation given by the authors of Ref. [7] itself, their analysis is just semi-quantitative and here we are able to provide a more comprehensive description of the experiment, which can frame the measured signal within the global picture described above. The aim of this subsection is to relate the XNDD signal to the dipole-quadrupole magnetic tensors $F^{(i-)}(\mathrm{E} 1-\mathrm{E} 2) \quad(\mathrm{i}=1,2,3)$ introduced above, discuss the physical meaning of each term, and substantiate the description by calculating the XNDD through an abinitio numerical code. ${ }^{29}$

If we consider the crystal and magnetic structure given in Ref. [35], the total absorption signal at iron $\mathrm{K}$ edge is obtained as the average over the four Fe absorption sites in the unit cell, $\sigma \equiv \sum_{j=1,4} \sigma^{(j)}$, where $\sigma^{(j)}$ (Eq. (5)), can be expressed as:

$$
\sigma^{(j)}=4 \pi^{2} \alpha \hbar \omega \sum_{n}\left|\left\langle\psi_{n}^{(j)}|\hat{O}| \psi_{g}^{(j)}\right\rangle\right|^{2} \delta\left(\hbar \omega-\left(E_{n}-E_{g}\right)\right)
$$

According to Refs. [ 7, 35], the unit cell is orthorhombic, with an electric polarization along the screw $b$ axis, and a net magnetization, below $\mathrm{T}_{c}$, along the $c$ axis. 
In the experiment performed in Ref. [ 7] four absorption spectra were measured, all with the x-ray wavevector directed along the $a$-axis: in two cases the electric polarization was directed along the $c$-axis, and in two cases along the $b$-axis. For each of these two subcases, the magnetic moment was selected to be either parallel or antiparallel to the easy c-axis, by means of an applied external magnetic field. We label them as $\sigma_{c}\left(H^{+}\right)$, $\sigma_{c}\left(H^{-}\right), \sigma_{b}\left(H^{+}\right), \sigma_{b}\left(H^{-}\right)$. The two measured dichroic spectra are then defined as: $\sigma_{c} \equiv \sigma_{c}\left(H^{+}\right)-\sigma_{c}\left(H^{-}\right)$, and $\sigma_{b} \equiv \sigma_{b}\left(H^{+}\right)-\sigma_{b}\left(H^{-}\right)$.

Before discussing the calculated numerical spectra, shown in Fig. 2, we just push our analytical derivation as far as possible, in order to demonstrate that the only quantities involved in this kind of dichroism are the tensors $F^{(i-)}(\mathrm{E} 1-\mathrm{E} 2)(\mathrm{i}=1,2,3)$. First of all, from the definition of $\sigma_{c}$ and $\sigma_{b}$, it is clear that only magnetic terms can contribute to the dichroism, as the structural absorption is the same for both $H^{ \pm}$configurations. This selects just $\hat{T}$-odd contributions. It is convenient to choose the reference frame $x y z$ in such a way that the quantization axis $z$ coincides with the direction of the x-ray beam (the $a$ crystallographic direction, in the configuration of Ref. [7]), while $x$ is directed along the electric polarization ( $b$ axis) and $y$ along the magnetic moment ( $c$-axis). Even though the usually chosen reference frame is the one with the $z$ axis along the magnetization, ${ }^{7}$ the frame considered here has the advantage that tensor properties acquire their simplest form. In this frame the polarizations have components $\hat{\epsilon}=\hat{\epsilon}^{*}=(1,0,0)$ for $\sigma_{b}$-dichroism and $\hat{\epsilon}=\hat{\epsilon}^{*}=(0,1,0)$ for $\sigma_{c}$-dichroism. In both cases, parityeven magnetic terms of E1-E1 and E2-E2 origin do not contribute, because no one of the three $T_{q}^{(1-)}(\mathrm{E} 1-\mathrm{E} 1)$ and $T_{q}^{(1-)}(\mathrm{E} 2-\mathrm{E} 2)$ or of the seven $T_{q}^{(3-)}(\mathrm{E} 2-\mathrm{E} 2)$ has a polarization dependence of the kind $\epsilon_{\alpha}^{*} k_{\beta} \epsilon_{\alpha} k_{\beta}$.

Therefore the only allowed terms are time-reversal and parity-odd E1-E2 terms. As all four Fe-ions belong to equivalent crystallographic positions, it is possible to relate them one another by means of symmetry operations, in such a way that Eq. (6) can be re-written as:

$$
\sigma=\left(1+\hat{T} \hat{m}_{z}\right)\left(1+\hat{T} \hat{C}_{2 x}\right) \sigma^{(1)}
$$

where $\hat{T}, \hat{m}_{z}$ and $\hat{C}_{2 x}$ are, respectively, the operations of time-reversal, mirror orthogonal to $z$ and two-fold axis along $x$. These symmetry operations are intended to act on the $F_{q}^{(p)}$ tensors. As only time-reversal odd and inversion-odd quantities contribute and as $\hat{m}_{z} F_{q}^{(p)}=$ $\hat{I} \hat{C}_{2 z} F_{q}^{(p)}=-(-)^{q} F_{q}^{(p)}$ and $\hat{C}_{2 x} F_{q}^{(p)}=(-)^{p} F_{-q}^{(p)}, 36$ we finally get that the only allowed terms are those of the kind: $\left(1+(-)^{q}\right)\left(F_{q}^{(p)}-(-)^{p} F_{-q}^{(p)}\right)$. Thus, XNDD is sensitive to $F_{0}^{(1)}, F_{0}^{(3)}, F_{2}^{(2)}-F_{-2}^{(2)}$, and $F_{2}^{(3)}+F_{-2}^{(3)}$. It is interesting to note that, separately, the first two terms $\left(F_{0}^{(1)}\right.$ and $\left.F_{0}^{(3)}\right)$ were already identified as responsible for the magnetochiral dichroism $(\mathrm{M} \chi \mathrm{D})$ in Ref. $34]$, and the second two $\left(F_{2}^{(2)}-F_{-2}^{(2)}\right.$ and $\left.F_{2}^{(3)}+F_{-2}^{(3)}\right)$ for the non-reciprocal gyrotropy (NRG) in Ref. [ 14]. Their contribution to XNDD can be written as follows: $\sigma_{b}=\sigma_{M \chi D}+\sigma_{N R G}$ and $\sigma_{c}=\sigma_{M \chi D}-\sigma_{N R G}$, where $\sigma_{M \chi D} \propto-\frac{\sqrt{3}}{2 \sqrt{5}} F_{0}^{(1)}-\frac{1}{\sqrt{10}} F_{0}^{(3)} \equiv\left\langle t_{z}-\frac{1}{6} l_{z}^{2} t_{z}\right\rangle^{37}$ and $\sigma_{N R G} \propto \frac{1}{\sqrt{6}}\left(F_{2}^{(2)}-F_{-2}^{(2)}\right)+\frac{1}{\sqrt{3}}\left(F_{2}^{(3)}+F_{-2}^{(3)}\right) \equiv\left\langle\left(l_{x}^{2}-\right.\right.$ $\left.\left.l_{y}^{2}\right) t_{z}\right\rangle-$ c.c. This allows us to disentangle the two contributions as: $\sigma_{M \chi D}=\left(\sigma_{b}+\sigma_{c}\right) / 2$ and $\sigma_{N R G}=\left(\sigma_{b}-\sigma_{c}\right) / 2$. A simple look at the experimental results of Ref. [7]) shows that the main contribution comes from $\sigma_{N R G}$, as $\sigma_{b}$ and $\sigma_{c}$ are almost opposite; however $\sigma_{M \chi D}$ is not zero. Notice that the irreducible tensors forming the $\mathrm{M} \chi \mathrm{D}$ signal both belong to the $\Gamma_{4}\left(T_{1}\right.$, in Bethe's notation) representation of the cubic group, while those of NRG belong to $\Gamma_{5}\left(\mathrm{~T}_{2}\right)$ : the former is invariant under $\hat{C}_{4}$-rotation, while the latter changes sign, since $l_{x}^{2} \leftrightarrow l_{y}^{2}$. This is the reason why they behave differently for $b$ and $c$-dichroism.

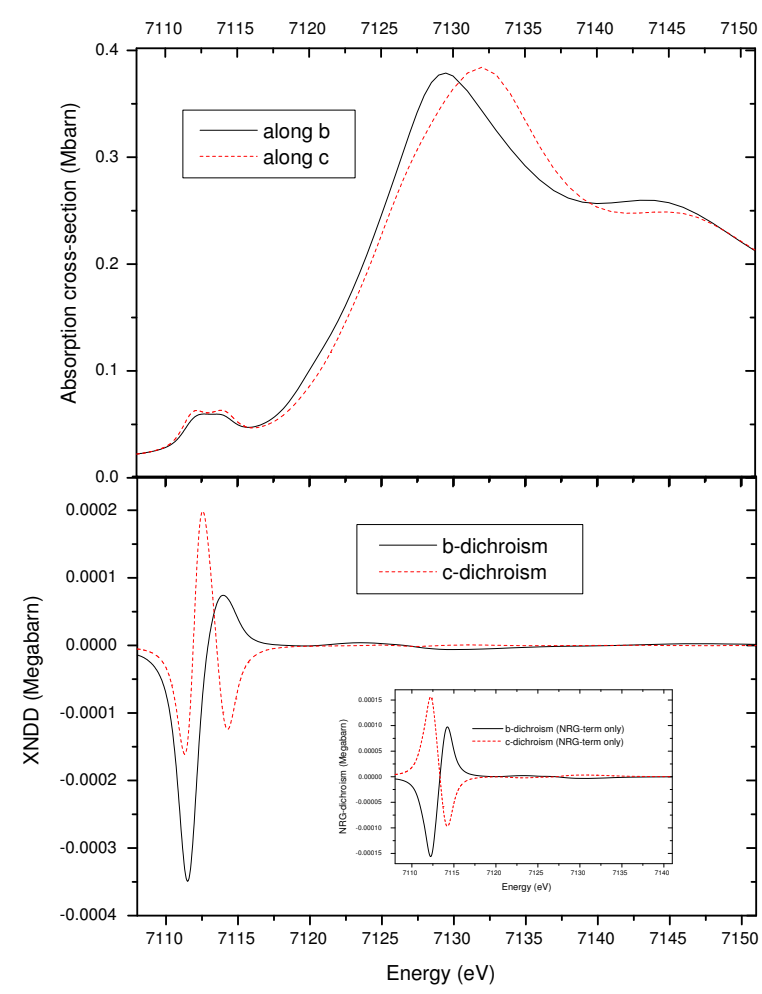

FIG. 2. Theoretical simulation of absorption spectrum and XNDD. The inset shows the contribution of $\sigma_{N R G}$ alone (see Conclusions).

Finally, in order to provide a link with the kind of density of states probed by the two dichroisms, we can evaluate the expectation values of $t_{z}-\frac{1}{6} l_{z}^{2} t_{z}$ and $\left(l_{x}^{2}-l_{y}^{2}\right) t_{z}-c . c$. and find: $\sigma_{M \chi D} \propto d_{x z}^{*} p_{x}+d_{y z}^{*} p_{y}-c . c$ and $\sigma_{N R G} \propto$ $d_{x z}^{*} p_{x}-d_{y z}^{*} p_{y}-c . c$, where $d_{\alpha \beta}\left(p_{\alpha}\right)$ are the (complex) coefficients of orbitals of $d_{\alpha \beta}\left(p_{\alpha}\right)$ type in the wave function. This picture is in complete agreement with the simpler analysis presented in Ref. [7]: in fact, from the previous expressions for $\sigma_{N R G}$ and $\sigma_{M \chi D}$, we get, for $b$ and $c$ dichroisms, $\sigma_{b} \propto d_{x z}^{*} p_{x}-c . c$. and $\sigma_{c} \propto d_{y z}^{*} p_{y}-c . c$. , which 
correspond to the transitions shown in their Fig. 4, mediated by the local polarization and spin-orbit, once the change in the axes labels is properly taken into account. Notice, however, that the orbital occupancy amplitude of empty states at pre-edge must be complex, in order to have a magnetic effect (the expectation value of magnetic operators on real orbitals is zero). Remarkably, the temperature dependence reported in Ref. 7, while showing that the XNDD signal follows the temperature profile of the magnetization, provides an indirect proof that polarization does not depend on temperature, as the XNDD signal is linear in the product of polarization and magnetization.

In order to substantiate these considerations, we have performed a numerical simulation with the ab initio magnetic code implemented in the fdmnes package. ${ }^{19,29}$ Our results basically confirm the previous picture, as shown in Fig. 2: the order of magnitude of the dichroism, slightly less than $10^{-2}$ of the absorption, correctly reproduces the experimental data. Moreover, the signal is entirely limited to the pre-edge, at the energy corresponding to the $3 d$ density of states, where hybridization of $p$ and $d$ orbitals is stronger: this latter seems to be a general feature of transition metal oxides, as inferred by other similar results. ${ }^{11,19,25}$

The agreement of our numerical simulation with experimental data, though quite good concerning the above features, is not completely satisfactory regarding the shape of the signal, especially for $\sigma_{c}$-dichroism. The reason is probably an overestimation of the $\mathrm{M} \chi \mathrm{D}$ signal, and is due to the fact that our numerical code is based on a single particle approach and cannot take correctly into account correlation effects, that are undoubtely strong in such a compound. Similar features were present already in the interpretation of RXS spectra in $\mathrm{V}_{2} \mathrm{O}_{3} \cdot{ }^{19}$ In spite of this, the fact that we are able to determine at least two of the main features of the XNDD signal, like its energy range and the relative magnitude dichroism/absorption, reveal that the main component of such a dichroism, ie, the NRG part, is determined by the crystal structure, which is correctly taken into account by our independent particle approach.

\section{CONCLUSIONS.}

In the first part of the paper we give a general classification, based on symmetry arguments, for various kinds of spectroscopies, in order to relate the corresponding observable quantities with each term of the electromagnetic multipole expansion in the material under study. In particular, we are able to interpret in this way x-ray natural circular dichroism as a measure of the non-magnetic $\vec{g}$ toroidal quadrupole, whereas, in the previous literature, ${ }^{6}$ it was ascribed to the operator $l_{z} t_{z}$.

Moreover, we have performed a numerical simulation of the absorption spectra of $\mathrm{GaFeO}_{3}$ to demonstrate the physical mechanism behind the XNDD. In this respect, a comment is required about the capability of our $a b$ initio magnetic code to deal with such tiny effects as the ones determined by parity and time-reversal odd multipoles, even though it is based on a single particle approach. As already stated above, we are able to determine quite well two main features of the XNDD signal, like its energy range and the relative magnitude dichroism/absorption. Moreover, as shown in the inset of Fig. 2, the NRG component of the dichroism alone reproduces quite well the experimental signal, which is mainly antisymmetric in the exchange $\sigma_{b} \leftrightarrow \sigma_{c}$, with a slight positive offset. Such a positive offset, determined by the $\mathrm{M} \chi \mathrm{D}$ part of the signal is not well reproduced by our numerical simulation. This seems to imply that the NRG term, roughly determined by the magnetic quadrupole of the system, is well reproduced by our independent particle approach, and, therefore, can be mainly ascribed to an effect of the crystal structure, whereas the $\mathrm{M} \chi \mathrm{D}$ component of the signal, which depends on the magnetic toroidal moment and octupole, is strongly affected by the electronic correlations and for this reason is badly reproduced by our single particle calculations.

${ }^{1}$ J.D. Jackson, Classical Electrodynamics, Ed. Wiley, 1975

${ }^{2}$ V.M. Dubovik and V.V. Tugushev, Phys. Rep. 187, 145 (1990)

${ }^{3}$ Ia.B. Zel'dovich, Zh. Eksp. Teor. Fiz. 33, 1531 (1957)[Sov. Phys. JETP 6, 1184 (1958)]

${ }^{4}$ A complete discussion about polar and axial toroidal moments in solid state physics can be found in Ref. [2].

${ }^{5}$ C.R. Natoli, Ch. Brouder, Ph. Sainctavit, J. Goulon, Ch. Goulon-Ginet, and A. Rogalev, Eur. Phys. J. B, 4, 1 (1998)

${ }^{6}$ P. Carra, R. Benoist, Phys. Rev. B 62, R7703 (2000)

${ }^{7}$ M. Kubota, T. Arima, Y. Kaneko, J.P. He, X.Z. Yu, and Y. Tokura, Phys. Rev. Lett. 92, 137401 (2004)

${ }^{8}$ The magnetic dipole term can be safely neglected in the $\mathrm{x}$-ray range.

${ }^{9} \mathrm{M}$. Blume, in Resonant Anomalous X-ray scattering, edited by G. Materlik, J. Sparks and K. Fisher (Elsevier, Amsterdam, 1994), p. 495.

${ }^{10}$ P. Carra, B.T. Thole, Rev. Mod. Phys. 66, 1509 (1994)

${ }^{11}$ S. Di Matteo, Y. Joly, A. Bombardi, et al., Phys. Rev. Lett. 91, 257402 (2003)

12 P. Carra, B.T. Thole, M. Altarelli, and X. Wang, Phys. Rev. Lett. 70, 694 (1993)

${ }^{13}$ P. Carra, A. Jerez, and I. Marri, Phys. Rev. B 67, 045111 (2003)

${ }^{14}$ S. Di Matteo, and C.R. Natoli, J. Synchrotron Radiat. 9, 9 (2002)

${ }^{15}$ V.E. Dmitrienko, E.N. Ovchinnikova, Acta Crys. A, 57, $642(2001)$

${ }^{16}$ I. Marri, and P. Carra, Phys. Rev. B 69, 113101 (2004) 
${ }^{17}$ B.T. Thole, P. Carra, F. Sette, G. van der Laan, Phys. Rev. Lett. 68, 1943 (1992)

${ }^{18}$ For RXS, the presence of the real anomalous term $f_{j}^{\prime}$ adds technical complications related to the inversion of KramersKrönig relations.

${ }^{19}$ Y. Joly, S. Di Matteo, and C.R. Natoli, Phys. Rev. B 69, $224401(2004)$

${ }^{20}$ Y. Joly, S. Grenier, and J. E. Lorenzo, Phys. Rev. B 68, 104412 (2003).

${ }^{21}$ S. Di Matteo, T. Chatterjee, Y. Joly, A. Stunault, J.A. Paixao, R. Suryanaryanan, G. Dhalenne, and A. Revcolevschi, Phys. Rev. B 68, 024414 (2003), and references therein.

${ }^{22}$ P. Santini, and G. Amoretti, Phys. Rev. Lett. 85, 2188 (2000)

${ }^{23}$ S.W. Lovesey, K.S. Knight, and D.S. Sivia, Phys. Rev. B 65, $224402(2002)$

${ }^{24}$ A. Bombardi, et al., unpublished

${ }^{25}$ S. Di Matteo, Phys. Rev. B 70, 165115 (2004)

${ }^{26}$ I.E. Dzjaloshinskii, Zh. Eksp. Teor. Fiz. 37, 881 (1959) [Sov. Phys. JETP 10, 628 (1960)]
${ }^{27}$ A. Astrov, Zh. Eksp. Teor. Fiz. 38, 984 (1960) [Sov. Phys. JETP 11,708 (1960)]

${ }^{28}$ I.M. Vitebskii, Sov. J. Low Temp. Phys. 7, 686 (1981).

${ }^{29}$ Y. Joly, Phys. Rev. B 63, 125120 (2001). The program can be downloaded at the web address http:/wwwcristallo.grenoble.cnrs.fr/simulation.

${ }^{30}$ S. Di Matteo, Y. Joly, C.R. Natoli, Phys. Rev. B 67, 195103 (2003)

31 J.P. Remeika, J. Appl. Phys. 31, 263 S (1960)

32 The transition temperature depends on the crystal growth, as shown, e.g., in Ref. [ 35].

${ }^{33}$ G.T. Rado, Phys. Rev. Lett. 13, 335 (1964)

${ }^{34}$ S. Di Matteo, and C.R. Natoli, Phys. Rev. B 66, 212413 (2002); see also Ref. [ 37].

35 T. Arima, D. Higashiyama, Y. Kaneko, J.P. He, T. Goto, S. Miyasaka, T. Kimura, K. Oikawa, T. Kamiyama, R. Kumai, and Y. Tokura, Phys. Rev. B 70, 064426 (2004)

${ }^{36}$ D.A. Varshalovich, A.N. Moskalev, V.K. Khersonskii, Quantum Theory of Angular Momentum, World Scientific, Singapore, 1988, pag. 142, Eqs. (6) and (8)

${ }^{37}$ Erratum to Phys. Rev. B 66, 212413, to be published 